\title{
Age and Gender Specific Thyroid Hormones and Their Relationships with Body Mass Index in a Large Chinese Population
}

\author{
Qianqian Song ${ }^{1}$, Xinxin Chen ${ }^{1}$, Yang $\mathrm{Su}^{2,3}$, Zhen Xie ${ }^{4,2}$, Shu Wang ${ }^{1}$ and Bin Cui ${ }^{1, *}$ \\ ${ }^{1}$ Shanghai Clinical Center for Endocrine and Metabolic Diseases, Shanghai Institute of Endocrine and Metabolic Diseases, Ruijin Hospital, Shanghai JiaoTong University \\ School of Medicine, Shanghai, China \\ ${ }^{2}$ Chinese Academy of Sciences Sichuan Translational Medicine Research Hospital, Chengdu, China \\ ${ }^{3}$ Clinical Laboratory, Sichuan Academy of Medical Sciences, Sichuan Provincial People's Hospital, Chengdu, China \\ ${ }^{4}$ Department of Dermatology, Sichuan Academy of Medical Sciences, Sichuan Provincial People's Hospital, Chengdu, China \\ "Corresponding author: Shanghai Clinical Center for Endocrine and Metabolic Diseases, Shanghai Institute of Endocrine and Metabolic Diseases, Ruijin Hospital, Shanghai \\ JiaoTong University School of Medicine, Shanghai, China. Email: bcui@sibs.ac.cn
}

Received 2018 January 19; Revised 2018 December 26; Accepted 2019 January 12.

\section{Abstract}

Objectives: To investigate the associations of thyroid-stimulating hormone (TSH), free triiodothyronine (FT3) and free thyroxine (FT4) with body mass index (BMI) and the effect of age and gender on these relationships in a large Chinese population.

Methods: A total of 97,997 individuals from a health examination center were selected. The medians (25th and 75th) of TSH, FT3 and FT4 were used to explore the trends based on gender, 10-year age groups and BMI. The relationships of TSH, FT3 and FT4 with BMI were assessed by generalized additive models (GAM) along with adjusting the effect of age and gender.

Results: After applying our exclusion criteria, 77,991 euthyroid participants (45,428 males and 32,563 females) were analyzed. The medians of TSH level based on BMI groups were significantly higher in female participants than those in males in all age groups $(\mathrm{P}<0.05)$, and the median FT3 level was lower in female subjects; however, there was no significant difference between male and female subjects in median FT4 level. The GAM analysis showed the non-linear positive association of TSH and FT3 with BMI, and these relationships were markedly influenced by age and gender. However, FT4 had a negative relationship with BMI, with neglectable effect of age and gender.

Conclusions: TSH, FT3 and BMI have a non-linear and positive quadratic relationship after age and gender adjustment. FT4, however, has a negative non-linear relationship with BMI with neglectable influence of age and gender.

Keywords: Thyroid-Stimulating Hormone, Free Triiodothyronine, Free Thyroxine, Body Mass Index, Generalized Additive Models

\section{Background}

Thyroid glands play an important role in the metabolism and growth of the human body by secreting thyroid hormones. Previous studies on thyroid-mediated regulation focused largely on patients with thyroid dysfunctions (1-4). Some studies have shown an association between thyroid hormone levels and body mass index (BMI), although the results are still controversial. In a 2006 study on 361 women and 40 men in the United Kingdom, Manji et al. reported lack of any relationship between either serum thyroid-stimulating hormone (TSH) or free thyroxine (FT4) level and BMI, since no difference was seen in the serum levels of either hormone between lean and obese subjects (5). In contrast, Sakurai et al. showed a significant positive relationship between serum TSH level and BMI among male subjects in a 2014 study on 1,044 men and 993 women in Japan (6). In contrast, Kitahara et al. found that TSH and FT3 were positively associated with BMI, while such an association did not exist for FT4 in neither gender in a study on 1623 men and 1491 women in the USA (7). Meng et al. reported a positive relationship between TSH level and BMI only among women in a study on 13,855 Chinese participants (8,532 men and 5,323 women) (8).

\section{Objectives}

To address this ambiguity, we collected data from a large euthyroid Chinese population (77,991 individuals aged 20 - 80 years) in an iodine sufficient area and analyzed the relationship between thyroid hormone levels and BMI, age and gender. 


\section{Methods}

\subsection{Study Population}

In this cross-sectional study, 97,997 individuals were recruited at the health examination center of Sichuan Provincial People's Hospital from October 2013 to April 2017. Essential demographic data like age, gender, height and weight and biochemical test results of TSH, FT3, FT4, thyroid peroxidase antibodies (TPOAb) and thyroglobulin antibodies (TgAb) were obtained from the patients' medical records. In addition, body mass index (BMI) was calculated as weight in kilograms divided by height in square meters.

The euthyroid (with no thyroid disorders) participants were identified using the following exclusion criteria: (1) age $<20$ years and $>80$ years, (2) serum FT4 or FT3 levels not within the reference range (FT4: 9.01-19.05 pmol/L and FT3: 2.63 - $5.70 \mathrm{pmol} / \mathrm{L})$,(3) serum TSH levels lower than 0.35 $\mathrm{mIU} / \mathrm{L}$ or above $4.94 \mathrm{mIU} / \mathrm{L}$ ( since such patients would have been under medical supervision and more likely to be ill, and (4) positive for thyroid antibodies (TgAb $>75 \mathrm{IU} / \mathrm{ml}$ and serum TPOAb $>30 \mathrm{IU} / \mathrm{m}$ ) to obviate any influence of these antibodies.

The study was approved by the Ethics Committee of Sichuan Provincial People's Hospital.

\subsection{Laboratory Measurements}

Standard laboratory quality evaluation procedures were used, and regular participation at inter-laboratory tests was also part of the quality control strategy. Serum TSH, FT3 and FT4 levels were measured by automated chemiluminescent immunoassays (Architect i2000SR; Abbott Laboratories, Chicago, US). The functional sensitivity of serum TSH was $0.0036 \mathrm{mIU} / \mathrm{L}$. The intra-assay coefficients of variation (CV) of serum TSH, FT4, FT3, TPOAb and $\mathrm{TgAb}$ were $1.3 \%$ - 6.3\%, and the inter-assay $\mathrm{CV}$ values were $2.0 \%-6.6 \%$. Our laboratory reference ranges provided by the manufacturer were as follows: TSH: 0.35 - $4.94 \mathrm{mIU} / \mathrm{L}$, FT4: 9.01 - $19.05 \mathrm{pmol} / \mathrm{L}$, FT3: 2.63 - $5.70 \mathrm{pmol} / \mathrm{L}, \mathrm{TPOAb}<30$ $\mathrm{IU} / \mathrm{mL}$ and $\mathrm{TgAb}<75 \mathrm{IU} / \mathrm{mL}$.

According to BMI, the participants were categorized into four groups, namely underweight with BMI 16 - 18.5 $\mathrm{kg} / \mathrm{m}^{2}$, normal weight at $18.5-24 \mathrm{~kg} / \mathrm{m}^{2}$, overweight at 24 $-28 \mathrm{~kg} / \mathrm{m}^{2}$ and obese at $28-35 \mathrm{~kg} / \mathrm{m}^{2}$. Age groups were categorized using 10-year intervals: 20 as 20 - 29 years old, 30 as 30 - 39 years old, 40 as 40 - 49 years old, 50 as 50 - 59 years old, 60 as 60 - 69 years old and 70 as $70-79$ years old.

\subsection{Statistical Analysis}

Continuous variables were tested for normality by Kolmogorov-Smirnov test using histogram and Q-Q plot. Data with normal and nearly normal distributions are presented as mean \pm standard deviation (SD), and those with non-normal distributions as median and inter-quartile range (IQR). The 25th and 75th percentiles were used for the definition of reference intervals and the medians. The 25th and 75th percentiles were calculated based on age/BMI groups in both genders. Independent $t$-test was used for parametric data and Wilcoxon rank sum test for nonparametric data when comparing age, BMI and TSH, FT3 and FT4 levels between men and women. The relationships between thyroid hormones and BMI with age and gender adjustment were generated using smoothing splines in generalized additive models (GAM) (9) by the "mgcv" package (version 1.8 - 24). All the statistical analyses were performed using R software (version 3.4.1) (10). P value less than 0.05 was considered statistically significant.

\section{Results}

Of the initial 97,997 participants, 12,568 (12.8\%) were positive for thyroid antibodies, $9.01 \%(8,830)$ had thyroid hormone levels beyond the reference range, $0.4 \%$ (397) had BMI outside 16 - $35 \mathrm{~kg} / \mathrm{m}^{2}$ and 1.07\% (1,054) were not between 20 - 80 years of age, and thus were excluded. The remaining 77,991 participants comprised of 45428 (58.25\%) males and 32563 (41.75\%) females (Table 1). The TSH and FT3 levels were significantly different between male and female participants $(\mathrm{P}<0.05)$, while there was no difference in FT4 level between the two genders.

\begin{tabular}{lccc}
\hline \multicolumn{4}{l}{ Table 1. Characteristics of the Study Population by Gender ${ }^{\mathrm{a}}$} \\
\hline & Women & Men & P Value \\
\hline Number & 32563 & 45428 & - \\
\hline Age, $\mathbf{y}$ & $41(31-50)$ & $44(34-52)$ & $<0.001$ \\
\hline BMI, $\mathbf{~ k g} / \mathbf{m}^{2}$ & $22.18 \pm 3.03$ & $24.53 \pm 3.09$ & $<0.001$ \\
TSH, $\mathbf{~ m I U} / \mathbf{L}$ & $2.20(1.57-2.99)$ & $1.89(1.36-2.58)$ & $<0.001$ \\
FT3, pmol/L & $4.43(4.09-4.78)$ & $4.39(4.75-5.10)$ & $<0.001$ \\
FT4, $\mathbf{~ p m o l} / \mathbf{L}$ & $12.69 \pm 1.35$ & $12.69 \pm 1.43$ & 0.810 \\
\hline
\end{tabular}

Abbreviations: BMI, body mass index; FT3, free triiodothyronine; FT4, free thyroxine; $\mathrm{TSH}$, thyroid-stimulating hormone.

${ }^{\mathrm{a}} \mathrm{P}<0.05$ is considered statistically significant.

Figure 1 exhibits the percentiles (median, 25th and 75th) of TSH, FT3 and FT4 in the different BMI groups and age categories among male and female subjects. The median TSH levels were obviously higher in female participants compared to males in each age group $(\mathrm{P}<0.05)$. The 
median FT3 levels were higher in males than in females before 60 years of age. However, the median FT4 levels showed no apparent difference between the two genders (Appendix 1-3 in Supplementary File).

Figure 2 and Table 2 present the relationships of TSH, FT3 and FT4 hormones with BMI before and after adjusting for age and gender by GAM analysis. The smoothing spline suggested a non-linear relationship between TSH level and BMI (Figure 2A, e.d.f $=2.804, \mathrm{P}<0.001$ ), with the TSH increasing after the inflection point of $25 \mathrm{~kg} / \mathrm{m}^{2}$. After adjusting for age and gender, TSH showed a quadratic correlation with BMI (Figure 2B, e.d.f $=1.705, \mathrm{P}<0.001$ ). FT3 and BMI had a positive relationship after the inflection point of $20 \mathrm{~kg} / \mathrm{m}^{2}$ without adjustment (Figure 2C, e.d.f $=6.118, \mathrm{P}$ $<0.001$ ). After age and gender adjustment, the relationship between FT3 and BMI was altered dramatically (Figure $2 \mathrm{D}$, e.d.f $=2.887, \mathrm{P}<0.001$ ). Regardless of age and gender adjustment, FT4 and BMI showed a significant non-linear relationship (Figure 2E, e.d.f $=6.049, \mathrm{P}<0.001$, Figure $2 \mathrm{~F}$, e.d.f $=6.030, \mathrm{P}<0.001)$.

\section{Discussion}

Our study aimed to determine the association of TSH, FT3 and FT4 with BMI in a large Chinese population. GAM revealed that after adjusting for age and gender, significant changes were seen pertaining to the relationship of TSH and FT3 with BMI, but not regarding FT4.

Several previous studies have investigated the relationship between TSH and BMI influenced by age and gender. In agreement with our findings, Svare et al. found a positive association between BMI and TSH in 9,954 women, but the association was slightly weaker in 5,066 men (11), Sakurai et al. found a significant relationship between TSH level and BMI exclusively in men (6), these studies indicated that sex could modify the association of BMI and TSH. However, Manji et al. found no evidence for the association between TSH and BMI using Spearman's rank correlation coefficient and the results were identical for 40 euthyroid men and 361 euthyroid women (5). In that study, the number of subjects was quite small and limiting the ability to detect any association.

For the relationship of serum FT3 level and BMI, Kitahara et al. performed a study of 1,623 euthyroid men and 1,491 euthyroid women and found that BMI was positively associated with FT3 level but not with FT4 level (12). Moreover, they reported an increase in FT3 level with every onequartile increase in BMI in men (1.0\%) and women (1.3\%), after adjusting for age and other factors. In our study, FT3 had an rising trend with increasing BMI in men and women, which was consistent with Kitahara et al. findings.

As it is well known, TSH is secreted in response to feedback from circulating thyroid hormone, thyroid hormone regulates body weight balance and body fat mass after its activation from the T4 to the active form T3 (13). A higher TSH concentration may be the result of a lower thyroid function (14), which may lead to a lower basal metabolic rate and obesity (15). However, other studies found that thyroid function and obesity may affect each other, such that adipose tissue secretes leptin, which contributes to the alterations in the secretion of thyrotropin-releasing hormone (TRH) and affects the secretion of TSH (16). Thus, future biomedical studies are needed to assess the relationship between thyroid system and obesity. Walrand et al. found FT3 increased energy expenditure in both young and the old rats, but old rats lost more weight than young ones (17). Ortega et al. found FT3 was associated with absolute and annual percentage of changes in weight $(\mathrm{r}=-0.27$, $\mathrm{P}=0.02)$ in 89 euthyroid Indians, and the association remained significant $(r=-0.28, P=0.009)$ after adjusting for age and gender (18). These findings were consistent with our results regarding the fact that the relationships of TSH and FT3 with BMI were influenced by gender and age.

The limitation of this study was that smoking status, ethnicity, alcohol intake, dietary habits, education, physical activity, menopause, insulin, which could modify the relationship between thyroid function and BMI, were not considered (7, 11, 12, 19-21). Compared to previous studies, our study was stronger on account of the large cohort of participants, which enabled us to determine the association between thyroid hormones and BMI relative to age and gender. In addition, GAM is a flexible and effective method to determine the nature of such relationships.

In conclusion, our findings suggested that TSH and FT3 had a positive non-linear relationship with BMI and their relationships remained strong after adjustment for age and gender. However, the non-linear negative relationship between FT4 and BMI was slightly effected by age and gender.

\section{Supplementary Material}

Supplementary material(s) is available here [To read supplementary materials, please refer to the journal website and open PDF/HTML]. 

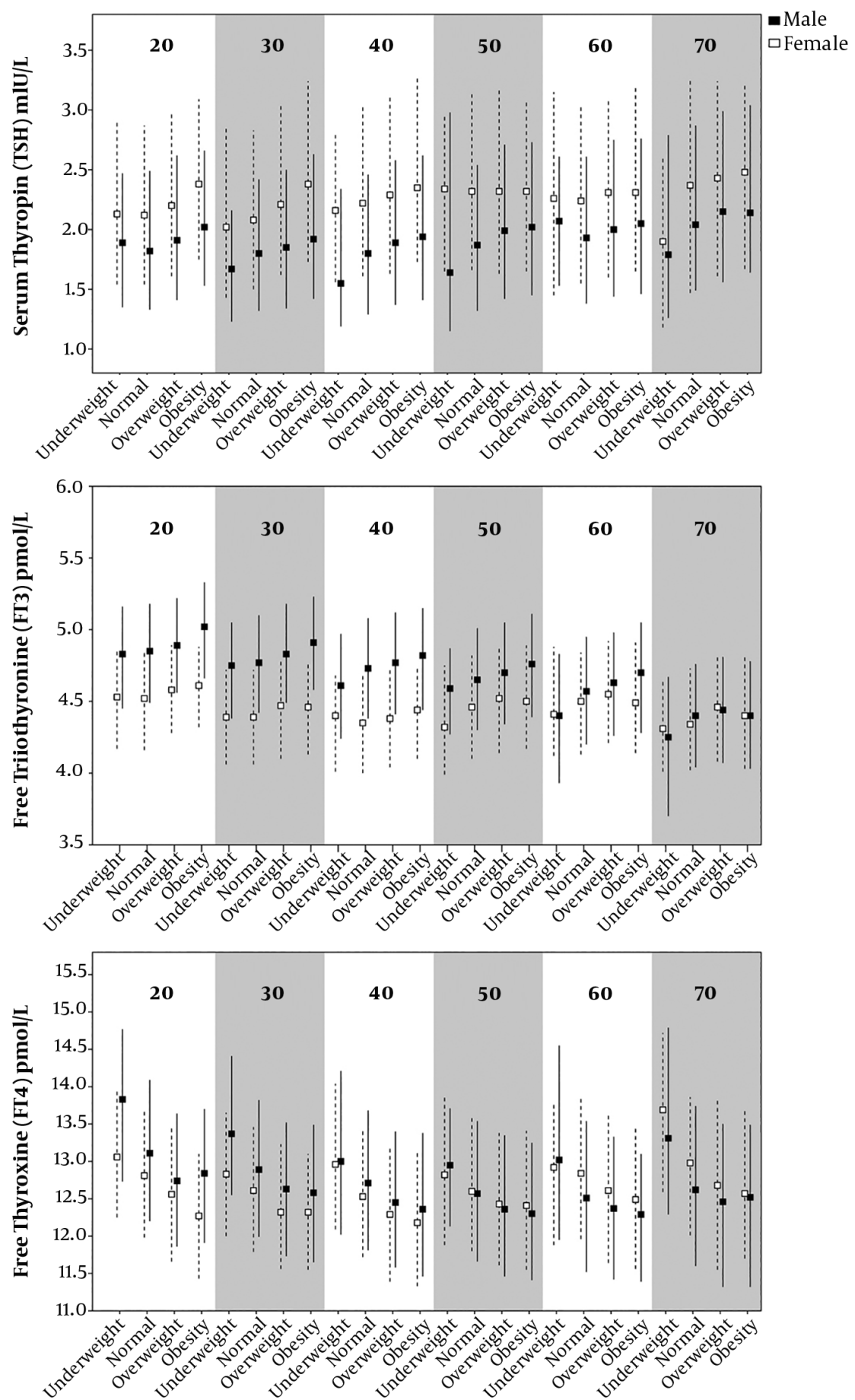

Figure 1. 25th, median, 75th Centiles of TSH (mIU/L), FT3 (pmol/L), FT4 (pmol/L) in age-BMI groups. Underweight: BMI within 16 - $18.5 \mathrm{~kg} / \mathrm{m}^{2} ;$ normal: BMI within 18.5 to 24 $\mathrm{kg} / \mathrm{m}^{2}$; overweight: BMI within $24-28 \mathrm{~kg} / \mathrm{m}^{2}$; obesity: BMI within $28-35 \mathrm{~kg} / \mathrm{m}^{2}$. The triangle and dashed line indicated female and square and solid line indicated male. 


\begin{tabular}{l} 
Table 2. The Result of the Generalized Additive Model on the Relationship of Body Mass Index with Thyroid Hormones ${ }^{\mathrm{a}, \mathrm{b}, \mathrm{c}}$ \\
\cline { 2 - 4 } \\
\cline { 2 - 4 }
\end{tabular}

A

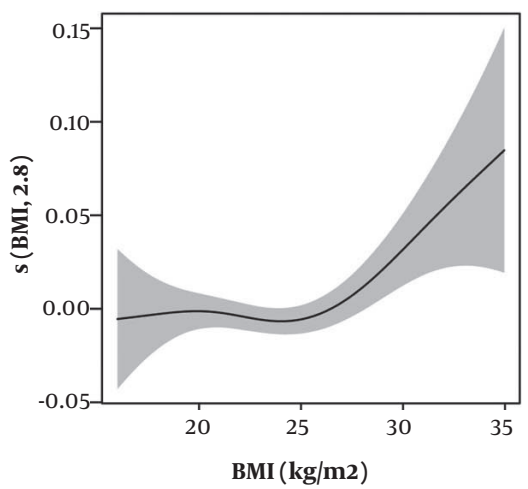

D

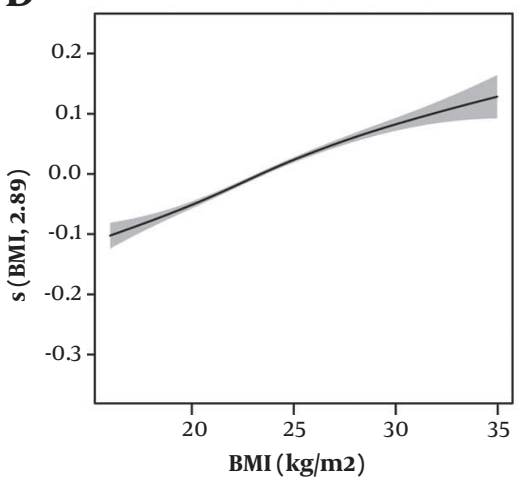

B

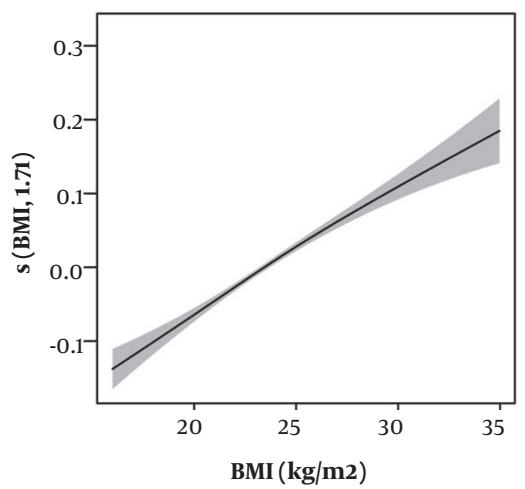

E

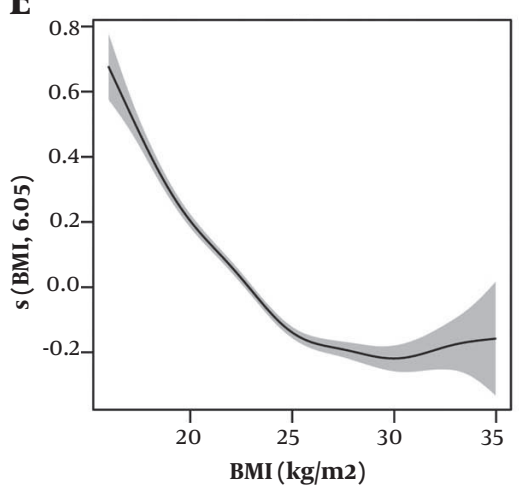

C

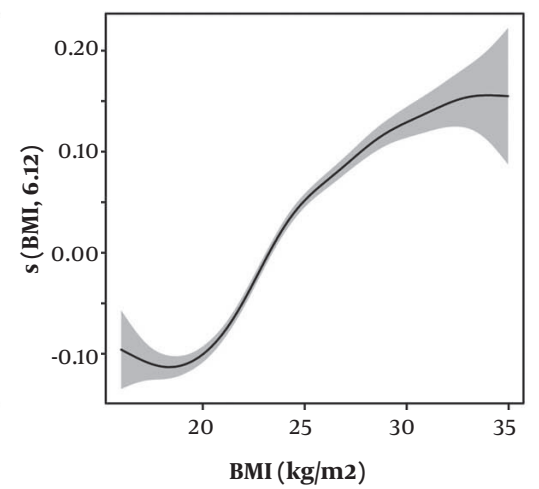

F

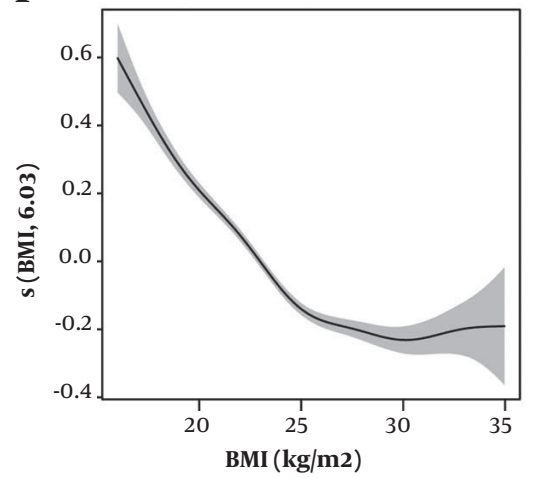

Figure 2. GAM assess the shape of relatioships between BMI $\left(\mathrm{kg} / \mathrm{m}^{2}\right)$ and thyroid hormones. A, relationship of TSH and BMI without adjustment; B, relationship of TSH and BMI with adjustment for age and sex; C, relationship of FT3 and BMI without adjustment; D, relationship of FT3 and BMI with adjustment for age and sex; E, relationship of FT4 and BMI without adjustment; F, relationship of FT4 and BMI with adjustment for age and sex. The shaded area of the trends are the $95 \%$ confidence intervals (95\%CI) of the fitted smoothing splines.

\section{Acknowledgments}

The study was partly supported by the National Key R\&D Program of China (2016YFC1305500), National Natural Science Foundation of China (No. 81670799, 81570707) and Shanghai Municipal Education Commission-Gaofeng Clinical Medicine Grant (No. 20152502).

\section{Footnotes}

Authors' Contribution: Study concept and design: Qianqian Song and Xinxin Chen; acquisition of data: Yang Su and Zhen Xie; analysis and interpretation of data: Qianqian Song and Xinxin Chen; drafting of the manuscript: Qianqian Song and Xinxin Chen; critical revision of the manuscript for important intellectual content: Bin Cui; statistical analysis: Qianqian Song; administrative, techni- 
cal, and material support: Yang Su, Zhen Xie and Bin Cui; study supervision: Bin Cui and Shu Wang. Qianqian Song and Xinxin Chen contributed equally to this work.

Conflict of Interests: No financial interest was related to the study.

Ethical Considerations: The study was approved by the Ethics Committee of Sichuan Provincial Peoples Hospital (2017-156, 2017-141).

Funding/Support: The study was partly supported by National Key R\&D Program of China (2016YFC1305500), National Natural Science Foundation of China (No. 81670799, 81570707) and Shanghai Municipal Education Commission-Gaofeng Clinical Medicine grant (No. 20152502).

\section{References}

1. De Pergola G, Ciampolillo A, Paolotti S, Trerotoli P, Giorgino R. Free triiodothyronine and thyroid stimulating hormone are directly associated with waist circumference, independently of insulin resistance, metabolic parameters and blood pressure in overweight and obese women. Clin Endocrinol (Oxf). 2007;67(2):265-9. doi: 10.1111/j.13652265.2007.02874.x. [PubMed: 17547687].

2. Torun E, Ozgen IT, Gokce S, Aydin S, Cesur Y. Thyroid hormone levels in obese children and adolescents with non-alcoholic fatty liver disease.JClin Res PediatrEndocrinol.2014;6(1):34-9. doi:10.4274/Jcrpe.1155. [PubMed: 24637308]. [PubMed Central: PMC3986737].

3. Wang CY, Chang TC, Chen MF. Associations between subclinical thyroid disease and metabolic syndrome. Endocr J. 2012;59(10):911-7. doi: 10.1507/endocrj.EJ12-0076. [PubMed: 22785370].

4. Kc R, Khatiwada S, Deo Mehta K, Pandey P, Lamsal M, Majhi S. Cardiovascular risk factors in subclinical hypothyroidism: A case control study in nepalese population. J Thyroid Res. 2015;2015:305241. doi: 10.1155/2015/305241. [PubMed: 26523236]. [PubMed Central: PMC4615208]

5. Manji N, Boelaert K, Sheppard MC, Holder RL, Gough SC, Franklyn JA. Lack of association between serum TSH or free T4 and body mass index in euthyroid subjects. Clin Endocrinol (Oxf). 2006;64(2):125-8. doi: 10.1111/j.1365-2265.2006.02433.x. [PubMed:16430708].

6. Sakurai M, Nakamura K, Miura K, Yoshita K, Takamura T, Nagasawa SY, et al. Association between a serum thyroid-stimulating hormone concentration within the normal range and indices of obesity in Japanese men and women. Intern Med. 2014;53(7):669-74. doi: 10.2169/internalmedicine.53.1387. [PubMed: 24694474].

7. Nyrnes A, Jorde R, Sundsfjord J. Serum TSH is positively associated with BMI.Int JObes (Lond).2006;30(1):100-5. doi: 10.1038/sj.ijo.0803112. [PubMed: 16189501].

8. Meng Z, Liu M, Zhang Q, Liu L, Song K, Tan J, et al. Gender and age impacts on the association between thyroid function and metabolic syndrome in Chinese. Medicine (Baltimore). 2015;94(50). e2193. doi: 10.1097/MD.0000000000002193. [PubMed: 26683929]. [PubMed Central: PMC5058901].

9. Hastie T, Tibshirani R. Generalized additive models. Stat Sci. 1986;1(3):297-310. doi:10.1214/ss/1177013604.

10. Ihaka R, Gentleman R. R: A language for data analysis and graphics. J Comput Graph Stat. 1996;5(3):299-314. doi 10.1080/10618600.1996.10474713.

11. Svare A, Nilsen TI, Bjoro T, Asvold BO, Langhammer A. Serum TSH related to measures of body mass: Longitudinal data from the HUNT Study, Norway. Clin Endocrinol (Oxf). 2011;74(6):769-75. doi 10.1111/j.1365-2265.2011.04009.x. [PubMed: 21521278].

12. Kitahara CM, Platz EA, Ladenson PW, Mondul AM, Menke A, Berring ton de Gonzalez A. Body fatness and markers of thyroid function among U.S. men and women. PLoS One. 2012;7(4). e34979. doi: 10.1371/journal.pone.0034979. [PubMed: 22511976]. [PubMed Central: PMC3325258].

13. Kim B. Thyroid hormone as a determinant of energy expenditure and the basal metabolic rate. Thyroid. 2008;18(2):141-4. doi: 10.1089/thy.2007.0266. [PubMed: 18279014]

14. Garcia-Solis P, Garcia OP, Hernandez-Puga G, Sanchez-Tusie AA, SaenzLuna CE, Hernandez-Montiel HL, et al. Thyroid hormones and obesity: a known but poorly understood relationship. Endokrynol Pol. 2018;69(3):292-303. doi: 10.5603/EP.2018.0032. [PubMed: 29952420].

15. Knudsen N, Laurberg P, Rasmussen LB, Bulow I, Perrild H, Ovesen $\mathrm{L}$, et al. Small differences in thyroid function may be important for body mass index and the occurrence of obesity in the population. $J$ Clin Endocrinol Metab. 2005;90(7):4019-24. doi: 10.1210/jc.2004-2225. [PubMed: 15870128].

16. Betry C, Challan-Belval MA, Bernard A, Charrie A, Drai J, Laville M, et al. Increased TSH in obesity: Evidence for a BMI-independent association with leptin. Diabetes Metab. 2015;41(3):248-51. doi 10.1016/j.diabet.2014.11.009. [PubMed: 25541439].

17. Walrand S, Short KR, Heemstra LA, Novak CM, Levine JA, CoenenSchimke JM, et al. Altered regulation of energy homeostasis in older rats in response to thyroid hormone administration. FASEB $J$ 2014;28(3):1499-510. doi: 10.1096/fj.13-239806. [PubMed: 24344330]. [PubMed Central: PMC3929673].

18. Ortega E, Pannacciulli N, Bogardus C, KrakoffJ. Plasma concentrations of free triiodothyronine predict weight change in euthyroid persons. Am J Clin Nutr. 2007;85(2):440-5. doi: 10.1093/ajcn/85.2.440. [PubMed: 17284741]. [PubMed Central: PMC1857361].

19. Souza Ade M, Sichieri R. Relationship between body mass index and thyrotropin in euthyroid women: Differences by smoking, race and menopausal status. Obes Facts. 2011;4(2):175-9. doi: 10.1159/000327843. [PubMed: 21577025].

20. Mehran L, Amouzegar A, Delshad H, Azizi F. Association between serum TSH concentration and body mass index in euthyroid subjects: The role of smoking. Arch Iran Med. 2012;15(7):400-3. [PubMed: 22724874].

21. Chaker L, Korevaar TI, Medici M, Uitterlinden AG, Hofman A, Dehghan A, et al. Thyroid function characteristics and determinants: The rotterdam study. Thyroid. 2016;26(9):1195-204. doi: 10.1089/thy.2016.0133. [PubMed: 27484151]. 